\title{
Eidgenössische Wahlen 2015
}

In den eidgenössischen Wahlen vom 18. Oktober 2015 entscheiden die Schweizer Stimmberechtigten, wer sie für die kommenden vier Jahre im Parlament politisch vertritt. Die FMH setzt sich dafür ein, dass die Ärzteschaft ihre Standpunkte und Lösungsvorschläge in den politischen und gesetzgeberischen Prozessen einbringen kann. Nachfolgend stellen wir Ihnen die FMH-Mitglieder vor, die für den National- oder Ständerat kandidieren und ab Herbst 2015 die ärztlichen Anliegen im Bundeshaus vertreten wollen.

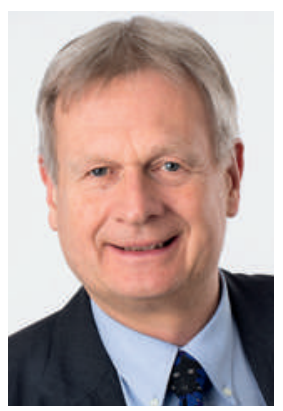

Aargau

\section{Ulrich Bürgi, 1957, Aarau, Aargau}

- Nationalratskandidat FDP

- Facharzt für Anästhesiologie, Facharzt für Intensivmedizin, Fähigkeitsausweis Klinische Notfallmedizin, Fähigkeitsausweis Notarzt

- Chefarzt am Zentrum für Notfallmedizin des Kantonsspitals Aarau

- Aargauischer Ärzteverband

- Mitglied des Aargauer Grossen Rates (seit 2014), ehemaliges Mitglied des Einwohnerrats von Aarau (2002-2013)

- Mitglied der Geschäftsleitung des Aargauischen Ärzteverbandes (seit 2014), Mitglied des Vorstandes des Bezirksärzteverbandes Aarau (seit 2002), Mitglied der Kommission eHealth der FMH (seit 2013), Mitglied des Vorstandes von eHealth Aargau (seit 2015), ehemaliger Präsident der Schweizerischen Gesellschaft für Notfall- und Rettungsmedizin (2009-2015)

- Keine Interessenbindungen
- www.fdp-ag.ch

- ulrich.buergi[at]ksa.ch

Viele Ärztinnen und Ärzte beobachten die Entwicklung unseres Gesundheitswesens mit Sorge. Die Schweiz wendet pro Jahr 11\% ihrer Wirtschaftsressourcen für das Gesundheitswesen auf. Damit sind wir Ärztinnen und Ärzte Teil einer bedeutenden nationalen Wirtschaftsproduktion. Doch welchen Stellenwert haben wir, wenn wichtige gesundheitspolitische Entscheidungen getroffen werden? Wir, die aufgrund unserer umfassenden Aus- und Weiterbildung die Verantwortung für die Gesundheitsversorgung tragen, sind zum Spielball von Ökonomen, Paramedizinern, Beratern, Investoren, Versicherungskonzernen und Lobbyisten geworden. Die Ärzteschaft muss wieder Stärke und Geschlossenheit zurückgewinnen, sie muss ein wichtiger nationaler Entscheidungsfaktor bleiben. Aber sie darf nicht dauernd reagieren, sie muss politisch agieren!

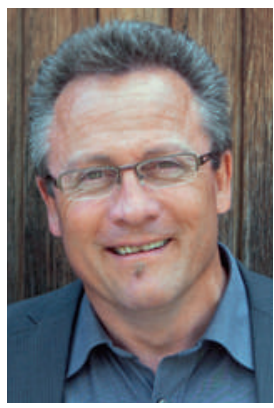

Bern
Daniel Beutler-Hohenberger, 1962, Gwatt bei Thun, Bern

- Nationalratskandidat Eidgenössische Demokratische Union (EDU)

- Facharzt für Allgemeine Innere Medizin

- Hausarzt in Thun, Publizist, Drogenexperte, Initiator des Pilotprojekts ANR (Accelerated Neuroregulation) - Opiatentzug im Spital Interlaken

- Ärztegesellschaft des Kantons Bern

- Mitglied des Berner Grossen Rates (ab September 2015), Redaktionskommission EDU-Standpunkt, Mitglied des Initiativkomitees «Schutz vor Sexualisierung in Kindergarten und Primarschule», ehemaliger Generalsekretär der «Arbeitsgemeinschaft Evangelischer Ärzte der Schweiz»

- Keine Interessenbindungen

- www.daniel-beutler-hohenberger.ch, http://daniel-beutler-hohenberger.politnetz.ch/
- www.facebook.com/\#!/daniel.beutler.921 (Facebook)

- dan.beutler[at]hin.ch

Christliche Werte sind an sich kein politisches Programm, aber sie verpflichten zu einer menschlichen Politik in Verantwortung vor dem Schöpfer und der Schöpfung. Christliche Werte sind nicht populär, aber sie geben Antworten auf die brennenden Fragen unserer Zeit, ohne zu dramatisieren und ohne zu beschönigen. Die unantastbare Menschenwürde ist die Basis jeder funktionierenden Gesellschaft. Das uneingeschränkte Recht auf Leben am Lebensanfang und -ende ist nicht verhandelbar.

Ich setze mich ein für neue Wege in der Drogenpolitik. Substitution und Legalisierung suchterzeugender Substanzen sind auf die Dauer nicht zielführend.

Eine gesunde Demut vor dem Wunder des Lebens lässt uns erkennen, dass sich das Menschsein nicht durch mechanistische Prinzipien allein erklären lässt. 


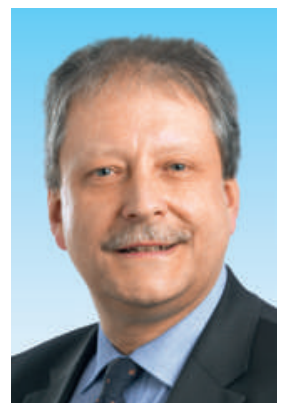

Hans-Peter Kohler, 1960, Spiegel bei Bern, Bern

- Nationalratskandidat FDP

- Facharzt für Innere Medizin

- Professor für Innere Medizin (Prof. Dr. med.), Klinikdirektor und Chefarzt der Klinik für Innere Medizin Tiefenau der Spital Netz Bern AG

- Ärztegesellschaft des Kantons Bern

- Mitglied des Berner Grossen Rates (seit 2014), Mitglied der kantonalen Gesundheits- und Sozialkommission Bern (GSoK) des Grossen Rates, Mitglied des Gemeindeparlaments von Köniz, Fraktionspräsident der FDP Köniz

- Vorstand der Ärztegesellschaft des Kantons Bern (BEKAG), Vorstand des Ärztlichen Bezirksvereins Bern Regio (ABV)

- Interessenbindungen: Stiftungsrat von Berner Gesundheit (BEGES) und des Berner Reha Zentrums Heiligenschwendi, Verwaltungsrat der SRO AG, Generalsekretär der Internationalen Gesellschaft für Innere Medizin
- www.hanspeter-kohler.ch

- hanspeter.kohler[at]spitalnetzbern.ch

Nebst der Spitzenmedizin muss eine qualitativ hochstehende medizinische Grundversorgung auch zukünftig ambulant wie auch stationär gewährleistet sein. Hierzu gehören der Erhalt und die Stärkung der hausärztlichen Grundversorgung und die Schaffung günstiger Rahmenbedingungen, unter welchen der ärztliche Nachwuchs nachhaltig gesichert bleibt. Dies bedingt die Neuevaluation des Numerus clausus, aber auch die Überprüfung, wie viele und welche Fachärzte zukünftig benötigt werden. Im Weiteren müssen die KVG-widrigen interkantonalen Wettbewerbsverzerrungen zwischen den Spitälern rasch behoben werden. Ich bin überzeugt, dass ich aus meinem beruflichen, privaten, standes- und parteipolitischen Umfeld viele konstruktive Anstösse und Lösungen sowie die dazu notwendige Erfahrung in den Nationalrat einbringen kann.

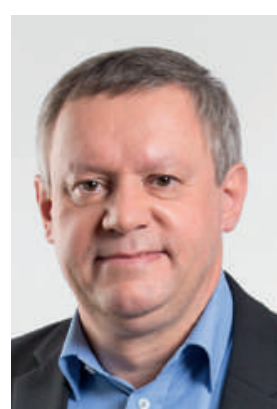

Jura
Pierre-Alain Fridez, 1957, Fontenais, Jura (sortant)

- Conseiller national PS (depuis 2011)

- Spécialiste en médecine générale

- Cabinet médical à Fontenais

- Société médicale du Jura

- Député du Parlement jurassien (depuis 2011), ancien maire de la commune de Fontenais (1997-2008)

- Membre de la commission politique de santé MFE

- Aucun lien d'intérêts

- pafmabe[at]bluewin.ch, pierre-alain.fridez[at]parl.ch

Dans un monde qui change, qui a érigé en dogmes les principes de libéralisme et de concurrence et qui voit les inégalités s'accroître, l'avenir de la santé pose question avec des aspects contradictoires toujours plus dif- ficiles à concilier. D’un côté un développement technologique et thérapeutique incessant, impressionnant, des prestations médicales toujours plus pointues et performantes, avec comme corollaire une explosion des coûts. De l'autre, une population vieillissante qui a en fait surtout besoin de soins de base, des primes qui s'envolent et deviennent une charge insupportable et des coupes budgétaires au niveau des collectivités publiques.

Dans ce contexte difficile, mes priorités sont simples: assurer un accès aux mêmes soins pour toutes et tous, assurer un financement socialement supportable de la santé et maintenir le filet de notre protection sociale. Renforcer le rôle central du médecin de premier recours en collaboration avec tous les acteurs de la santé et continuer à espérer que le temps de la caisse publique viendra... 


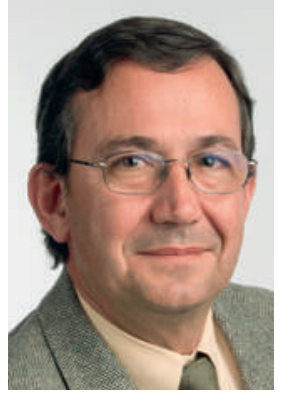

Neuchâtel
Blaise Courvoisier, 1955, La Chaux-de-Fonds, Neuchâtel

- Candidat au Conseil national et au Conseil des Etats, UDC

- Spécialiste en chirurgie

- Médecin KFOR

- ASMAC, Société Neuchâteloise de Médecine

- Ancien député au Grand Conseil neuchâtelois (20052013)

- Ancien membre du comité de la Société Neuchâteloise de Médecine

- Lien d'intérêts: président de la clinique de la Tour à Chaux-de-Fonds (1992-2013)

- https://www.facebook.com/udcne?fref=ts (Facebook)

- blaise.courvoisier[at]hotmail.ch
Pour tenter de résoudre le problème récurrent de la pénurie médicale de généralistes et de certains spécialistes, il faut revoir entièrement la formation médicale et postgraduée en créant des écoles professionnelles de médecine, séparées de l'Université, redimensionner à la baisse les hôpitaux universitaires pour ne leur laisser que les cas lourds et complexes et planifier, au niveau fédéral, une couverture d'hôpitaux généralistes, sièges de ces écoles professionnelles de médecine. La formation universitaire, spécialisée et postgraduée, sera, elle, destinée à un petit pourcentage d'étudiants choisis au cours de leur formation de base.

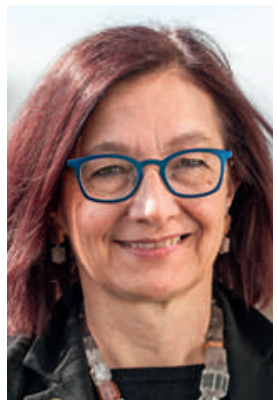

St. Gallen

\section{Yvonne Gilli, 1957, Wil, St. Gallen}

(bisher)

- Nationalrätin GPS (seit 2007)

- Fachärztin für Allgemeine Innere Medizin mit den Schwerpunkten Gynäkologie und Komplementärmedizin

- Fachärztin für Allgemeine Innere Medizin in selbstständiger Praxistätigkeit

- Ärztegesellschaft des Kantons St.Gallen

- Mitglied der Kommission für soziale Sicherheit und Gesundheit (seit 2008) sowie der Finanzkommission (seit 2011), Mitglied der Kommission für Wissenschaft, Bildung und Kultur (bis 2011), Mitglied der Geschäftsleitung Grüne Kanton St. Gallen (seit 2007)

- Interessenbindungen: Mitbegründerin und Verwaltungsrätin des Ärztenetzwerkes xundart AG, Präsidentin Patientenstelle Ostschweiz, Beirätin reliva Patientenhotel AG und der Vereinigung der Rehabilitationskliniken der Schweiz

- www.yvonne-gilli.ch
- https://www.facebook.com/yvonne.gilli (Facebook), https://twitter.com/\#!/YvonneGilli (Twitter) - yvonne.gilli[at]parl.ch

Der Ärzteschaft droht im Bundeshaus die Rolle des Sündenbockes. Es braucht das direkte standespolitische Engagement: fair, kompromissbereit und aus einer starken standespolitischen Identität heraus. Das gelingt nur, wenn die verschiedenen Fachgesellschaften geschlossener auftreten gegenüber der Politik und zeigen, dass die Gesundheit der Patienten und Patientinnen im Zentrum ihres Engagements steht. Die Partizipation an politischen Prozessen braucht einen langen Atem, eine gute Vernetzung und strategisches Handeln. Die nächste Legislatur wird wichtig! Die Tarifrevision und die zunehmende Konkurrenz um beschränkte Mittel werden uns herausfordern, ökonomisch und ethisch. Ich werde dazu beitragen, dass die Ärzteschaft nicht berufsfremd reguliert und durch Interessenkonflikte zerrieben wird. 


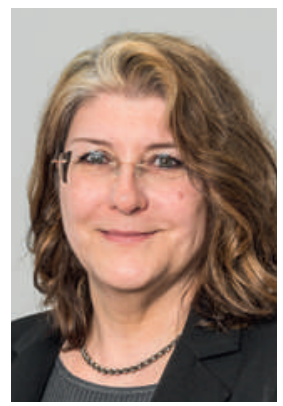

Veronika Breitler-Voigt, 1957, St. Gallen,

\section{St. Gallen}

- Nationalratskandidatin BDP

- Fachärztin für Psychiatrie und Psychotherapie, Fachärztin für Psychosomatische Medizin (D)

- Niedergelassen in eigener Praxis in St.Gallen und Kirchberg

- Ärztegesellschaft des Kantons St. Gallen

- Keine Interessenbindungen

St. Gallen
- www.ostschweizerinnen.ch, www.bdp.info/sg

- www.drbreitler.ch, www.nogppm.ch

- veronika.breitler[at]tbwil.ch

Dafür engagiere ich mich in der BDP:

- Apropos Ärztemangel: einen landeseigenen Beitrag zur Ausbildung leisten, Studienmöglichkeiten für
Mediziner in der Ostschweiz mit einem eigenen Master-Studiengang einführen und ausbauen

- Möglichkeiten zu mehr Teilzeitarbeit für junge Mediziner anbieten, die die Facharztanerkennung, v.a. geschlechtsabhängig, nicht beeinträchtigen

- Gesundheitspolitik, u.a. mehr Eigenverantwortung der Bevölkerung für die eigene Gesundheit

- Sozialwerke: sinnvolle Wiedereingliederung nach IV

- Frauenfragen: Gender, Vereinbarkeit von Karriere und Life-Balance

- Familienpolitik: Familienthemen liegen mir am Herzen, da ich viel mit strukturell und institutionell verschachtelten Familienproblemen zu tun habe und an Lösungen arbeite

- Bildung und öffentliche Sicherheit

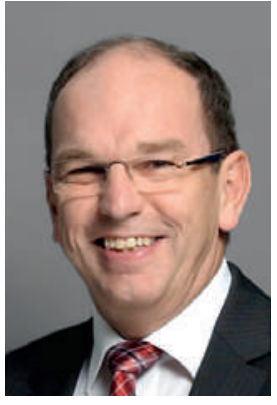

St. Gallen

\section{Bruno Damann, 1957, Gossau, St. Gallen}

- Nationalratskandidat CVP

- Facharzt für Allgemeine Innere Medizin

- Arzt mit eigener Praxis, Stadtrat von Gossau (SG)

- Ärztegesellschaft des Kantons St.Gallen

- Stadtrat von Gossau (SG) (Departementsvorsteher Jugend Alter Soziales, seit 2013), Mitglied des St.Galler Kantonsrats (seit 2012),

- Interessensbindungen: Verwaltungsratspräsident des Ärztehauses Lerchenstrasse AG und der House of PORT AG, Verwaltungsrat der Sana Fürstenland AG und der Walter Zoo Gossau AG, Mitglied des Advisory Board der HCI AG (Tochter der Galenica)

- www.bruno-damann.ch

- https://www.facebook.com/bruno.damann (Facebook)

- bruno.damann[at]hin.ch
Das Gesundheitswesen der Schweiz gilt als eines der besten der Welt. Diesem Niveau gilt es Sorge zu tragen. Vor allem aber muss es bezahlbar bleiben. Darum muss die Hausarztmedizin wieder ins Zentrum der Gesundheitsversorgung rücken. Es müssen mehr Allgemeinmediziner ausgebildet werden. Notwendig ist eine kompetente politische Vertretung mit der Bereitschaft zur Zusammenarbeit.

Als Initiator einer grösseren Gemeinschaftspraxis habe ich mir als Ziel gesetzt, Kosten zu optimieren, die Kräfte zu bündeln und die Kompetenzen optimal zum Nutzen der Patienten einzusetzen. Ich setze mich für die Eigenständigkeit der Ärzte ein. Eine Staatsmedizin wäre ein grosser Verlust für die Schweizer Bevölkerung. Aus eigener Erfahrung vertraue ich dabei auf eine möglichst schlanke Regeldichte des Staates. 


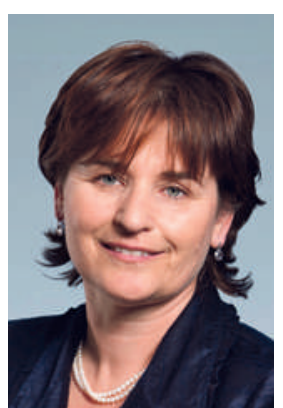

Marina Carobbio Guscetti, 1966, Lumino, Ticino (finora)

- Consigliere nazionale PSS (dal 2007)

- Specialista in medicina generale

- Medico

- Ordine dei Medici Grigioni

- Membro della commissione della sicurezza sociale e della sanità (dal 2011), della commissione delle finanze (dal 2007) e della delegazione delle finanze

Ticino (dal 2009), vicepresidente PS Svizzero (dal 2008), deputata in Gran Consiglio del Ticino (1991-2007)

- Legami d'interessi: presidente dell'Associazione Svizzera Inquilini, presidente della coordinazione nazionale delle dipendenze (NAS-CPA), copresidente dell'Associazione aiuto medico al Centro America

- www.marinacarobbio.ch, https://www.facebook.com/pages/ Marina-Carobbio (pagina Facebook)

- https://twitter.com/MarinaCarobbio (Twitter), https://www.facebook.com/marina.carobbio (profilo Facebook)

- marina.carobbio[at]bluewin.ch, marina.carobbio[at]parl.ch

Negli ultimi decenni la Svizzera ha fatto grandi progressi, sia a livello di politica sociale che a livello di quella sanitaria (AVS, assicurazione malattia obbligatoria, assicurazione invalidità e molto altro). Progressi che favoriscono la solidarietà e le pari opportunità e che rafforzano, quindi, la coesione sociale nel nostro paese. Oggi, purtroppo, questi progressi vengono nuovamente messi in discussione: il mio lavoro parlamentare, commissionale e il mio impegno politico anche al di fuori delle istituzioni politiche è dedicato al mantenimento e a un ragionato adeguamento delle assicurazioni sociali e a un sistema sanitario pubblico di qualità e accessibile a tutti.

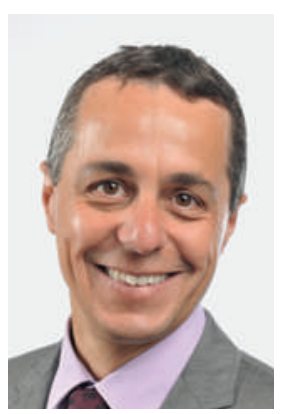

Ticino
Ignazio Cassis, 1961, Collina d'Oro, Ticino (finora)

- Consigliere nazionale PLR. I liberali radicali (dal 2007)

- Specialista in prevenzione e salute pubblica, specialista in medicina interna

- Presidente di quattro organizzazioni nazionali (CURAVIVA Svizzera, Radix, curafutura, EQUAM), incaricato d'insegnamento alle università di Berna, Lausanne, Lugano e Zurigo

- Società medica cantonale di Berna

- Membro (dal 2007) e vicepresidente (dal 2013) della commissione della sicurezza sociale e della sanità, consigliere comunale di Collina d'Oro (dal 2004 al 2014)

- Vicepresidente della FMH (dal 2008 al 2012), vicepresidente dell'ASMAC (dal 1992 al 1996), presidente dell'ASMACT (dal 1989 al 1992)

- Legami d'interessi: Presidente di Fourchette verte Ticino, membro di comitato del Forum Managed Care
- www.ignaziocassis.ch

- https://www.facebook.com/pages/IgnazioCassis/486301424808499 (Facebook), https://twitter.com/ignaziocassis (Twitter)

- ignazio.cassis[at]parl.ch

Grazie al mio percorso professionale ho osservato il sistema sanitario da diverse angolazioni: come medico clinico e di salute pubblica, come medico cantonale, dall'angolo dei fornitori di prestazioni e da quello degli assicuratori. Questa pluralità di prospettive mi è preziosa nel lavoro quotidiano di politica sanitaria nazionale. Mi aiuta a non smarrirmi nella giungla del sistema sanitario tra i mille interessi divergenti e, soprattutto, a non perdere di vista l'obiettivo centrale: assicurare agli abitanti della Svizzera la miglior assistenza medica possibile, in un sistema sanitario solidale e dal finanziamento sostenibile. Al centro del sistema c'è il paziente, che per me liberale-radicale è un cittadino responsabile. M'impegno da sempre per lo sviluppo sostenibile del nostro sistema sanitario solidale e fondato sulla concorrenza. 


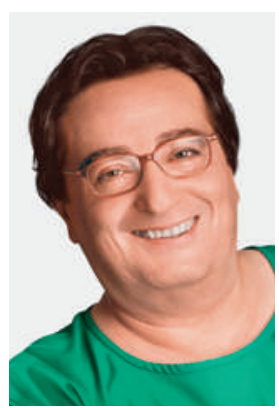

Ticino

\section{Franco Denti, 1957, Lugano, Ticino}

- Candidato al Consiglio nazionale I Verdi del Ticino

- Specialista in medicina generale

- Medico

- Deputato in Gran Consiglio del Ticino

- Presidente dell'Ordine dei Medici del Cantone Ticino

- Nessun legame d'interessi

- www.francodenti.ch

- http://twitter.com/denti_franco (Twitter), https://www.facebook.com/pages/FrancoDenti/678613185617565?fref=ts (Facebook)

- franco.denti[at]bluewin.ch
Sono un medico di famiglia, presidente dell'Ordine dei Medici del Cantone Ticino, membro di diversi gremii che a livello federale si occupano di sanità e di socialità. Il mio obiettivo è affrontare problematiche che toccano da vicino i cittadini-pazienti, dalla spirale dei premi delle casse malati che in Ticino sono da sempre troppo elevati. Uno studio fatto fare all'OMCT ha calcolato che dal 1996 al 2013 le casse malati hanno formato riserve eccedentarie per oltre 400 milioni. Desidero difendere il mercato del lavoro, il territorio e la salvaguardia delle bellezze storiche e le tradizioni del nostro amato paese.

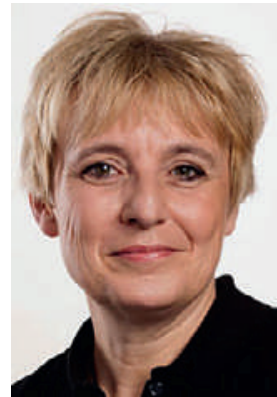

Vaud

\section{Brigitte Crottaz, 1957, Epalinges, Vaud}

- Candidate au Conseil national, PS

- Spécialiste en médecine interne et en endocrinologie-diabétologie

- Pratique en cabinet privé

- Société Vaudoise de Médecine

- Conseillère communale à Epalinges (depuis 2005), députée au Grand Conseil vaudois (depuis 2012), membre de la commission de santé du Grand Conseil (depuis 2012)

- Membre du comité (trésorière) de la section des endocrinologues praticiens suisses, membre du comité unitaire de l'initiative «Pour le remboursement des soins dentaires»

- Aucun lien d'intérêts

- www.ps-vd.ch

- Facebook: Brigitte Crottaz

- bcrottaz[at]bluewin.ch
Le fossé qui sépare les classes aisées et défavorisées est en constante augmentation. Ceci s'observe dans divers domaines, dont celui de la santé. Malgré le souhait louable à l'origine de la LAMal, son système basé sur la concurrence conduit à des écarts toujours plus grands entre les personnes en bonne santé, qui concluent des contrats d'assurance "low cost», et celles atteintes dans leur santé, qui doivent opter pour des contrats plus coûteux, ayant la double pénalité de la maladie et du coût.

Le système actuel ne peut perdurer sans des réformes qui passeront par un examen à Berne où le lobby des assurances a montré sa toute puissance. Mon souhait est donc de défendre, à un niveau où cela est possible, un système de santé juste et équitable, sans dérive vers une médecine à deux vitesses. 


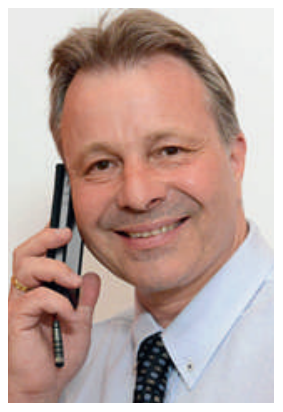

Vaud

\section{Thierry Dubois, 1961, Gilly, Vaud}

- Candidat au Conseil national, UDC

- Spécialiste ORL

- Spécialiste ORL indépendant, médecin agréé à l'Hôpital de la Tour, à la Clinique de la Colline et à la Clinique de Genolier

- Société Vaudoise de Médecine

- Municipal à Gilly, président de l'UDC du district de Nyon, membre UDC du Comité Central Vaud et Suisse

- Ancien médecin consultant à l'HCUG

- Aucun lien d'intérêts

- www.thierry-dubois.ch

- https://www.facebook.com/thierry.dubois.771 (Facebook)

- thierrydubois[at]bluewin.ch
Nous devons être fiers de notre système de santé qui est probablement un des meilleurs au monde et qui a fait ses preuves. Mais nous devons avant tout nous préoccuper de la santé de nos patients, préserver leurs intérêts et leurs droits; notamment celui de choisir leur médecin. Il faut garantir à tous un accès à des soins de qualité et de proximité en renforçant la collaboration entre les différents acteurs de santé.

Nous avons de plus en plus de devoirs, mais nous avons aussi des droits et nous devons les faire valoir en participant activement, notamment à Berne, à l'élaboration de solutions qui tiennent compte des intérêts des patients et de tous les professionnels de la santé. La santé de la Suisse c'est ma priorité, nous représenter c'est mon devoir!

Pour lire la suite: www.thierry-dubois.ch

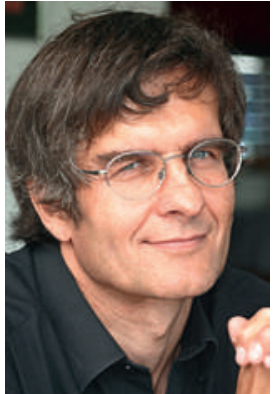

Vaud

\section{Blaise Vionnet, 1956, Echallens, Vaud}

- Candidat au Conseil national, Parti Vert'libéral

- Spécialiste en médecine générale et en médecine tropicale et des voyages

- Médecin généraliste installé à Echallens (depuis 1994)

- Société Vaudoise de Médecine, Médecins de famille Vaud

- Aucun lien d'intérêts

- http://www.votezpournous.ch/

- https://www.facebook.com/blaise.vionnet (Facebook), https://twitter.com/b_vionnet (Twitter)

- ab.vionnet[at]gmail.com

Mon engagement en politique de santé se concentrera sur les réformes suivantes afin de limiter l'explosion des coûts:
1. Favoriser une médecine de proximité (accessibilité à tous, désengorgement des services d'urgences, maintien des personnes âgées à domicile, augmentation des places d'études).

2. Privilégier une concentration de la médecine hautement spécialisée dans quelques centres de référence.

3. Euvrer à davantage de transparence à tous les niveaux de notre système de santé. Cette transparence doit permettre une amélioration de la qualité, telle qu'une meilleure coordination dans la chaîne de soins ou par une baisse des complications diverses.

4. Promouvoir la prévention quaternaire à l'aide de l'approche de Smarter Medicine (less is more). 


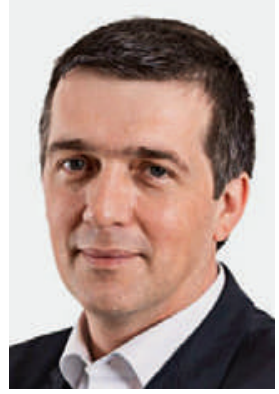

\section{Patrick Hildbrand, 1971, Brig-Glis, Wallis}

- Nationalratskandidat SVP

- Facharzt für Kardiologie

- Privatsprechstunde in der Herzpraxis Brig, Co-Chefarzt am Walliser Herzzentrum der Clinique de Valère in Sitten, Konsiliararzt an der Luzerner Höhenklinik in Montana

- Walliser Ärztegesellschaft

- Stadtrat Brig-Glis (Verantwortlicher fürs Ressort Wallis Bau und Planung, seit 2013), Mitglied des Walliser Grossen Rates für die SVPO-Fraktion (seit 2013)

- Präsident der Oberwalliser Ärztegesellschaft (20112013), Präsident der Walliser Kardiologiegesellschaft (2008-2013)

- Keine Interessenbindungen

- www.miishaerz.ch

- https://www.facebook.com/miishaerz (Facebook)

- miishaerz[at]herzpraxis-brig.ch
Mit grossem Aufwand konnten wir gemeinsam die Abschaffung der freien Arztwahl (Managed Care) und die absolute Kontrolle durch eine Einheitskasse abwenden. Diese Erfahrung hat uns gezeigt, wie wichtig es ist, die gesundheitspolitischen Entscheide vor Ort in Bundesbern zu beeinflussen.

Ich setzte mich mit Überzeugung gegen die Verstaatlichung der ambulanten Medizin und die weitere Zentralisierung der stationären Grundversorgung ein. Ich lehne jegliche Rationalisierungen medizinischer Leistungen $a b$, die unter dem Deckmantel der Kostenreduktion laufen und eine Zweiklassenmedizin fördern.

Zudem bin ich überzeugt, dass die qualitativ hochstehenden Ausbildungsplätze im Gesundheitsbereich in der Schweiz ausgebaut werden müssen.

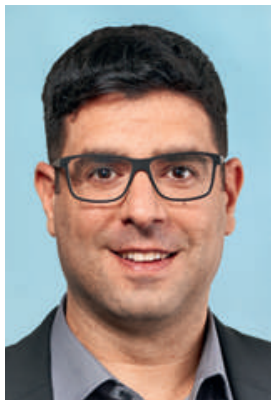

Zürich

\section{Angelo Barrile, 1976, Zürich, Zürich}

- Nationalratskandidat SPS

- Facharzt für Allgemeine Innere Medizin

- Hausarzt

- VSAO, Ärztegesellschaft des Kantons Zürich

- Mitglied des Zürcher Kantonsrats (seit 2010), Mitglied der kantonsrätlichen Kommission für soziale Sicherheit und Gesundheit (seit 2011)

- Mitglied der Geschäftsleitung des VSAO Zürich

- Interessenbindungen: Mitglied des Vorstandes der kispex Kinder-Spitex Kanton Zürich und der Sozialvernetzten Lebensgemeinschaft (SLG)

- www.barrile.ch

- www.facebook.com/nrbarrile (Facebook), www.twitter.com/anbarrile (Twitter)

- angelo[at]barrile.ch
Die Gesundheit der Schweizer Bevölkerung hat ihren Preis! Ich will die medizinische Qualität ins Zentrum der gesundheitspolitischen Diskussion rücken. Deshalb setze ich mich ein für:

- die Sicherung des langfristigen Bedarfs an Ärztinnen und Ärzten. Dafür sollen endlich genügend Studienplätze in Medizin bereitgestellt werden. Die gute Aus- und Weiterbildung muss gewahrt bleiben. Eine bürokratische Zulassungssteuerung ohne erwiesene Wirkung auf die Kosten lehne ich ab. Sie bremst lediglich den ärztlichen Nachwuchs aus.

- die öffentlichen Spitäler. Diese sind für mich Grundpfeiler des Service Public und dürfen nicht privatisiert werden.

- die Hausarztmedizin. Sie muss wieder attraktiver werden. Dazu gehört neben einer fairen Entlöhnung auch ein besseres Angebot an flexiblen Teilzeitstellen. 


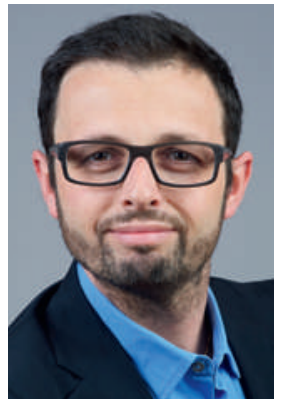

Zürich

\section{David Garcia, 1975, Zürich, Zürich}

- Nationalratskandidat Alternative Liste

- Facharzt für Psychiatrie und Psychotherapie mit Schwerpunkt Konsiliar- und Liaisonpsychiatrie, Sexualtherapeut (Deutsche Gesellschaft für Sexualforschung)

- Manager mit Schwerpunkt Gender-Varianz in der Klinik für Plastische, Rekonstruktive, Ästhetische und Handchirurgie des Universitätsspitals Basel

- Ärztegesellschaft des Kantons Zürich, Schweizerische Gesellschaft für Psychiatrie und Psychotherapie (SGPP), Schweizerische Gesellschaft für Konsiliar- und Liaisonpsychiatrie (SSCLP), VSAO

- Beratungsexperte für den Europarat in Fragen zur sexuellen Orientierung, Geschlechtsidentität und Menschenrechte, ehemaliges Mitglied der Ständigen Kommission für Weiter- und Fortbildungsfragen der SGPP

- Keine Interessenbindungen
- www.al-zh.ch

- dagar[at]doctor.com

Als Konsiliarpsychiater in einem Universitätsspital kenne ich sowohl die somatische wie auch die psychiatrische Welt. In dieser Brückenbauerfunktion habe ich gesehen, wie die DRG-Logik vielerorts das medizinische Denken zu Gunsten eines ökonomischen Herumagierens verdrängt hat. Die knappen Ressourcen werden in Prestigebauten und in der Administration investiert. Die Patientinnen und Patienten sowie das medizinischpsychiatrische Personal haben das Nachsehen.

Ich will mich für ein modernes, ganzheitliches Denken in den medizinischen Institutionen einsetzen. Wir brauchen ein effizientes öffentliches Gesundheitssystem, welches nicht nur über Profite nachdenkt, sondern sich ernsthaft um die Gesundheit der Schwächsten und vermehrt um die Aus- und Weiterbildungsqualität zukünftiger Kolleginnen und Kollegen ernsthaft kümmert.

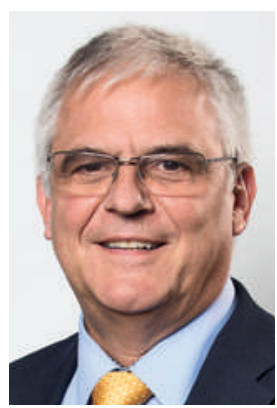

Zürich

\section{Josef Widler, 1954, Zürich, Zürich}

- Nationalratskandidat CVP

- Facharzt für Allgemeine Medizin

- Haus-, Familien- und Heimarzt

- Ärztegesellschaft des Kantons Zürich

- Mitglied des Zürcher Kantonsrats (seit 2014), Mitglied des Gemeinderats der Stadt Zürich (2005-2011)

- Präsident der Ärztegesellschaft des Kantons Zürich (seit 2015), Mitglied des Vorstands der Ärztegesellschaft des Kantons Zürich (2008-2014), Präsident des erfolgreichen Initiativkomitees «Wahlfreiheit beim Medikamentenbezug» (2006)

- Keine Interessenbindungen

- www.josef-widler.ch

- Josef.Widler[at]bluewin.ch
Als Präsident der grössten kantonalen Ärztegesellschaft möchte ich in Bern die Interessen der Ärzteschaft vertreten und mich für eine vernünftige Gesundheitspolitik einsetzen. Dabei muss das Wohl der Patientinnen und Patienten und dürfen nicht die Kosten im Zentrum der Gesundheitspolitik stehen. Die hohe Lebenserwartung wird die Nachfrage nach ärztlichen Leistungen ansteigen lassen. In Zukunft werden noch höhere Ansprüche an die fachlichen und menschlichen Kompetenzen der Ärzteschaft gestellt. Ärztinnen und Ärzte werden immer die letzte Verantwortung für die Behandlung der Patienten übernehmen müssen. Deshalb setze ich mich für die Ausbildung von genügend leistungswilligen, fachkompetenten und empathischen Ärztinnen und Ärzten ein. Die Politik muss Voraussetzungen schaffen, die unsere jungen Kolleginnen und Kollegen ermuntern, in eigener fachlicher und wirtschaftlicher Verantwortung den freien Arztberuf auszuüben.

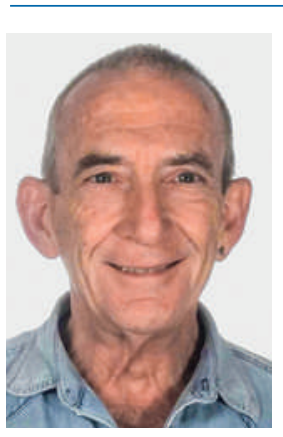

Zürich

\section{David Winizki, 1948, Zürich, Zürich}

- Nationalratskandidat Alternative Liste

- Facharzt für Allgemeine Medizin

- Hausarzt

- Ärztegesellschaft des Kantons Zürich, Vereinigung Unabhängiger ÄrztInnen VUA

- Keine Interessenbindungen

- david.winizki[at]bluewin.ch

Als Hausarzt werde ich oft konfrontiert mit der Verarmung breiter Bevölkerungsschichten, immer knauserigeren Sozialversicherungen, omnipotenteren Kran- kenkassen und einer zunehmenden Ökonomisierung des Gesundheitswesens. Dieses gehört jedoch zu jenen Gütern, die wir nicht zum Spass konsumieren, sondern zum Überleben brauchen. Es muss deshalb der ganzen Bevölkerung ohne Rationierungen zugänglich sein und solidarischer als heute bezahlt werden, am besten analog den AHV-/IV-Beiträgen und ohne die sinnlosen, manchmal gefährlich hohen Selbstbeteiligungen. Leistungserbringer sollen gut aus-, weiter- und fortgebildet werden, vernetzt arbeiten und adäquat bezahlt werden. Existenzbedingungen, die ein selbstbestimmtes Leben in Würde erlauben, sind als Gesundheitsvorsorge wichtiger als Birchermüesli und Joggen. 